\title{
ROLE OF ADIPONECTIN IN PATIENTS WITH NON-INSULIN DEPENDENT DIABETES MELLITUS
}

\author{
A. M. El-Nuweihy ${ }^{1}$, N. T. El-Melegy ${ }^{1}$, N. F. Ameen ${ }^{2}$ and E. M. Radwan ${ }^{1}$ \\ ${ }^{1}$ Department of Medicinal Biochemistry, ${ }^{2}$ Department of Internal Medicine, Faculty of \\ Medicine, Assiut University, Assiut, Egypt
}

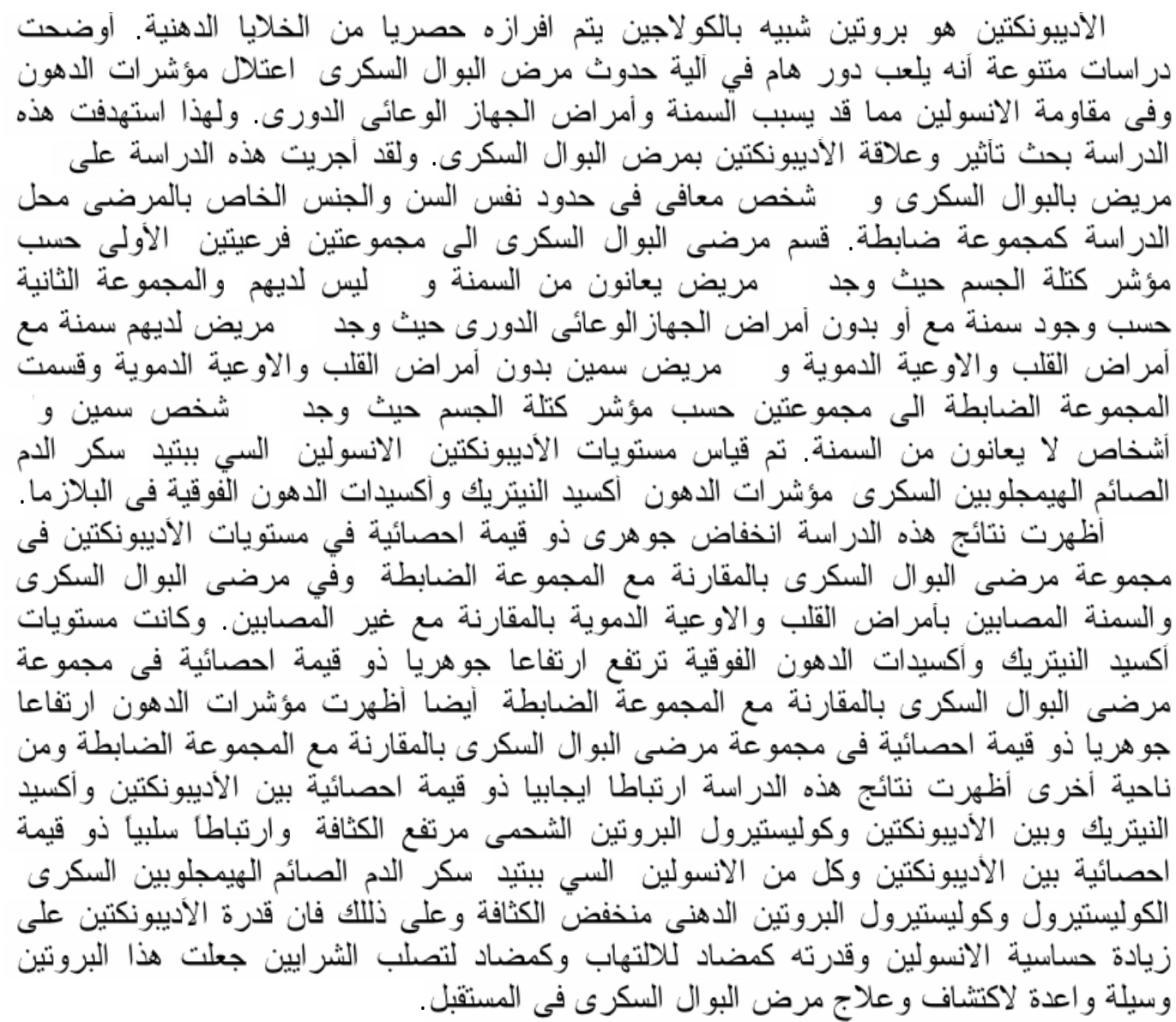

Adiponectin is a collagen-like protein that is solely secreted by adipocytes. Different studies showed that it plays an important role in the pathophysiology of insulin resistance, diabetes and dyslipidemia and thus affects risk for cardiovascular disease and obesity. In the present study the role of adiponectin in pathogenesis of type 2 diabetes mellitus was evaluated. The current study was carried on 51 diabetic patients with documented NIDDM and 22 age and sex matched healthy controls. Diabetic patients were subdivided into 2 subgroups according to BMI where 40 were obese and 11 were non obese and according to the presence of cardiovascular disease with obesity where 16 were obese with CVD and 24 were obese with no CVD. Controls were subdivided according to BMI where 7 were non obese and 15 were obese. The levels of plasma adiponectin, insulin, c-peptide, fasting blood glucose, glycated hemoglobin, lipid profile, NO and lipid peroxides. The results of the present study showed that adiponectin was significantly lower in all groups with variations compared to controls, in obese patients with CVD than those without CVD. NO and MDA levels were higher in diabetic patients than in controls and the highest levels of MDA were observed in patients with cardiovascular disease. Lipid profile was altered in diabetic patients showing higher levels than in controls. In the diabetic patients, adiponectin was significantly positively correlated with NO and HDL, while it was significantly negatively correlated with glucose, HbAlC, Cholesterol, 
LDL, insulin and c-peptide. The ability of adiponectin to increase insulin sensitivity in conjunction with its anti-inflammatory and anti-atherogenic properties have made this novel adipocytokine a promising therapeutic tool for the future.

\section{INTRODUCTION}

Non-insulin dependent diabetes mellitus (diabetes mellitus type 2) is the most common form of diabetes. It is a disorder that is characterized by high blood glucose. Unlike type 1 diabetes and other types of diabetes, type 2 diabetes has its own pathophysiological abnormalities, such as impaired insulin secretion, peripheral insulin resistance, and excessive hepatic glucose production. Along with diabetes type 2, obesity and cardiovascular diseases are very common in patients ${ }^{1}$. The association of obesity with development of type 2 diabetes may be partly mediated by altered secretion of adipokines by adipose tissues. Adipose tissue is a complex, essential, and highly active metabolic and endocrine organ. It does not only respond to afferent signals from traditional hormone systems and the central nervous system but also expresses and secretes factors with important endocrine functions. These factors include leptin, adiponectin, plasminogen activator inhibitor-1, proteins of the renin-angiotensin system, acylation stimulating protein and resistin $^{2}$.

Adiponectin is a collagen-like protein that is solely secreted by adipocytes. Accumulating evidences from animal and human studies demonstrate that adiponectin plays an important role in the pathophysiology of insulin resistance, diabetes, lipid metabolism and inflammation and thus affects risk for cardiovascular diseases ${ }^{3}$. It has been reported that reduction in plasma adiponectin level may be related to the elevation of insulin resistance and/or hyperinsulinemia associated with diabetes and that adiponectin deficiency contributes to the induction of insulin resistance ${ }^{4}$. However the association between adiponectin and insulin resistance have been controversial; whereas some investigators reported that negative significant correlation occurs between plasma adiponectin levels and fasting insulin level ${ }^{5 \& 6}$, these observations were not confirmed by other investigators ${ }^{7 \& 8}$.

Adiponectin was reported to mimic the vascular as well as the metabolic actions of insulin. It inhibits macrophage transformation to foam cells as well as their phagocytic activity ${ }^{9}$. In addition, adiponectin diminishes oxidized low density lipoproteins accumulation in blood vessel wall and increases nitric oxide production in endothelial cells ${ }^{10}$. It is suggested that adiponectin via these mechanisms may not only protect against atherosclerosis but also retard its progression ${ }^{11}$.

The ability of adiponectin to increase insulin sensitivity in conjunction with its antiinflammatory and anti-atherogenic properties have made this novel adipocytokine a promising therapeutic tool for the future ${ }^{12}$. Thus, we aimed to evaluate the role of adiponectin in the pathogenesis of type 2 diabetes mellitus through determining its level in the different studied subgroups of patients, to examine the relationship between adiponectin, glycemic control and lipid profile through determining its level in the different studied subgroups of patients and to assess the relationship between adiponectin and obesity as well as atherosclerosis-related cardiovascular diseases (CVD) in type 2 diabetes mellitus subjects.

\section{SUBJECTS AND METHODS}

The current study was carried on 51 diabetic patients with documented NIDDM and 22 age and sex matched healthy controls. The patients were selected from the outpatient clinic and inpatients of internal medicine department of Assiut university hospital.

Diabetes was diagnosed if the fasting blood glucose level was $\geq 126 \mathrm{mg} / \mathrm{dL}$ and plasma glucose was $\geq 200 \mathrm{mg} / \mathrm{dL}$ two hours after a $75 \mathrm{~g}$ oral glucose load as in a glucose tolerance test (OGTT) according to the WHO $(2007)^{13}$. Glycated hemoglobin concentration was done and normal level was considered below $6 \%{ }^{14}$.

Formal consents were obtained from patients and controls. The study was approved by Ethical committee of Faculty of Medicine.

Patients with the following conditions were excluded:

- Smoking habits. 
- Any kind of malignancy.

- Essential hypertension.

- Liver or kidney disorders.

Each patient was subjected to complete thorough medical history and clinical examinations and a resting electrocardiogram was performed for assessment of ischemic heart diseases. The height and weight were recorded for all patients and controls and presented in the form of body mass index (BMI) (Quetelet index) (weight in $\mathrm{Kg} /$ height in $\left.\mathrm{m}^{2}\right)$.

Diabetic patients were subdivided into 2 subgroups:

- First subgroup was divided according to presence of cardiovascular disease and obesity where 16 patients were considered obese and had cardiovascular diseases while 24 patients were obese and free of C.V.D.

- Second subgroup divided with respect to BMI according to (WHO), BMI $\geq 25$ was considered overweight and 230 was obese where 11 patients had BMI $<25$ while 40 patients had $\mathrm{BMI} \geq 25$.

Controls were subdivided according to BMI including 7 non obese and 15 obese subjects.

After an overnight fast, $7 \mathrm{ml}$ venous blood was drawn from an antecubital vein under aseptic conditions in the early morning, each blood sample was divided into 2 tubes containing EDTA: the first tube was refrigerated at $2-8^{\circ} \mathrm{C}$ for $1-2$ days for the assay of glycated hemoglobin, while the second tube was directly centrifuged at $5000 \mathrm{rpm}$ for 10 min. then the separated plasma was divided into 2 aliquots and stored at $-70^{\circ} \mathrm{C}$ till the time of assay of adiponectin, insulin, c-peptide, nitric oxide and lipid profiles (total cholesterol, HDL-cholesterol, LDL-cholesterol and triglycerides), glucose was determined immediatly.

Adiponectin was measured according to the method described by $\mathrm{Hu}^{15}$. by an AviBion Human Adiponectin (Acrp30) ELISA kit, supplied from Ani Biotech Orgenium Laboratories, Business Unit Tiilitie, 3 FIN07120 Vantaa FINLAND. Insulin was measured, according to the method described by Flier ${ }^{16}$ by an ELISA kit cat. No. KAP1251 which was manufactured by BioSource Europe
S.A. C-peptide was determined according to the method described by Eastham ${ }^{17}$ by an ELISA kit, product no. 2725-300, produced by Monobind Inc., Lake forest, USA. Glucose was determined according to method described by Trinder ${ }^{18}$ by Stanbio enzymatic glucose kit, procedure no. 1075 manifactured by Stanbio laboratory, Inc, San Antonio, Texas, USA, Glycated hemoglobin was determined by Stanbio Glycohemoglobin, procedure No. 0350 according to the method described by Abraham $^{19}$. Cholesterol was determined by Stanbio Liquicolor ${ }^{\mathrm{R}}$ Cholesterol kit, procedure No. 1010 according to Trinder ${ }^{20}$. HDL was determined by Stanbio HDL Cholesterol kit, procedure no. 0599 according to the method described by Finley ${ }^{21}$. LDL-Cholesterol was determined by a kit provided by Quimica Clinica Aplicada S.A., Spain according to the method described by Assmann ${ }^{22}$. Trigycerides level was determined by Stanbio Liquicolor ${ }^{R}$ Triglycerides kit, procedure no. 2100-430 according to the method described by Wahlefeld $^{23}$. Nitric oxide was determined by Griess reaction described by Van Bezooijen ${ }^{24}$. Lipid peroxides (malondialdehyde) was determined by the thiobarbituric acid (TBA) assay according to Thayer ${ }^{25}$.

Statistical analyses were performed using the SPSS statitistical program for analyzing the obtained data. All results are presented as mean \pm SD. $\mathrm{P}$ values $<0.05$ were considered significant. Correlations between variables were performed using spearman rank correlation coefficient.

\section{RESULTS}

Table 1 shows the demographic and clinical characteristics of type 2 diabetic patients and control subjects, where significantly higher levels of fasting glucose $(p<0.001)$, glycated hemoglobin $(p<0.001)$, plasma cholesterol $(\mathrm{p}<0.001)$, triglycerides $(\mathrm{p}<$ $0.01)$ and LDL cholesterol $(\mathrm{p}<0.01)$ were found in patients than controls while plasma HDL cholesterol was significantly lower in patients than controls $(\mathrm{p}<0.05)$.

Table 2 shows levels of biochemical parameters in type 2 diabetic patients versus controls, where diabetic patients showed significantly higher plasma levels of insulin $(p<0.001)$, c-peptide $(p<0.01), \mathrm{NO}(\mathrm{p}<0.001)$ 
Table 1: The demographic and clinical characteristics of type 2 diabetic patients and controls.

\begin{tabular}{|c|c|c|c|}
\hline Parameter & Controls $n=22$ & Patients $n=51$ & Significance \\
\hline $\begin{array}{l}\text { Age (years) } \\
\text { Mean } \pm \text { SD } \\
\text { Range } \\
\end{array}$ & $\begin{array}{c}54.4 \pm 5.8 \\
40-60 \\
\end{array}$ & $\begin{array}{c}57.1 \pm 5.57 \\
39-66 \\
\end{array}$ & NS \\
\hline $\begin{array}{c}\text { BMI }\left(\mathrm{Kg} / \mathrm{m}^{2}\right) \\
\text { Mean } \pm \mathrm{SD} \\
\text { Range } \\
\end{array}$ & $\begin{array}{l}26.9 \pm 7 \\
21.45-40\end{array}$ & $\begin{array}{c}29.31 \pm 4.52 \\
22.5-44\end{array}$ & NS \\
\hline $\begin{array}{c}\text { Fasting glucose }(\mathrm{mg} / \mathrm{dl}) \\
\text { Mean } \pm \mathrm{SD} \\
\text { Range } \\
\end{array}$ & $\begin{array}{c}79.86 \pm 14.3 \\
49-100\end{array}$ & $\begin{array}{c}304.69 \pm 127.6 \\
110-610 \\
\end{array}$ & $\mathrm{p}<0.001$ \\
\hline $\begin{array}{c}\text { Glycated hemoglobin } \mathrm{HbA} 1 \mathrm{c} \% \\
\text { Mean } \pm \text { SD } \\
\text { Range } \\
\end{array}$ & $\begin{array}{c}5.26 \pm 0.36 \\
4.7-6.2 \\
\end{array}$ & $\begin{array}{c}9.07 \pm 2.04 \\
6.4-16.4 \\
\end{array}$ & $\mathrm{p}<0.001$ \\
\hline $\begin{array}{c}\text { Cholesterol }(\mathrm{mg} / \mathrm{dl}) \\
\text { Mean } \pm \mathrm{SD} \\
\text { Range } \\
\end{array}$ & $\begin{array}{c}117.85 \pm 38.49 \\
80-175 \\
\end{array}$ & $\begin{array}{c}168.90 \pm 58.71 \\
70-350 \\
\end{array}$ & $\mathrm{p}<0.001$ \\
\hline $\begin{array}{c}\text { Triglycerides }(\mathrm{mg} / \mathrm{dl}) \\
\text { Mean } \pm \mathrm{SD} \\
\text { Range }\end{array}$ & $\begin{array}{c}63.27 \pm 31.49 \\
10-108\end{array}$ & $\begin{array}{c}119.08 \pm 54.82 \\
46-275\end{array}$ & $\mathrm{p}<0.001$ \\
\hline $\begin{array}{c}\text { LDLcholesterol }(\mathrm{mg} / \mathrm{dl}) \\
\text { Mean } \pm \text { SD } \\
\text { Range }\end{array}$ & $\begin{array}{c}61.09 \pm 30 \\
10-105\end{array}$ & $\begin{array}{c}95.78 \pm 53.87 \\
10-215\end{array}$ & $\mathrm{p}<0.01$ \\
\hline $\begin{array}{c}\text { HDLcholesterol }(\mathrm{mg} / \mathrm{dl}) \\
\text { Mean } \pm \mathrm{SD} \\
\text { Range }\end{array}$ & $\begin{array}{c}47.23 \pm 12.53 \\
26-82\end{array}$ & $\begin{array}{c}40.63 \pm 10.69 \\
10-65\end{array}$ & $\mathrm{p}<0.05$ \\
\hline
\end{tabular}

Table 2: Plasma levels of biochemical parameters of type 2 diabetic patients and controls.

\begin{tabular}{|c|c|c|c|}
\hline Parameter & Controls $n=22$ & Patients $n=51$ & Significance \\
\hline $\begin{array}{c}\text { Insulin }(\mu \mathrm{IU} / \mathrm{mL}) \\
\text { Mean } \pm \text { SD } \\
\text { Range } \\
\end{array}$ & $\begin{array}{c}17.91 \pm 4.2 \\
9-25.5 \\
\end{array}$ & $\begin{array}{c}33.08 \pm 16.25 \\
5.5-85\end{array}$ & $\mathrm{p}<0.001$ \\
\hline $\begin{array}{l}\text { C-peptide }(\mathrm{ng} / \mathrm{ml}) \\
\text { Mean } \pm \text { SD } \\
\text { Range }\end{array}$ & $\begin{array}{c}1.62 \pm 0.74 \\
0.8-4\end{array}$ & $\begin{array}{c}2.52 \pm 1.31 \\
0.47-5\end{array}$ & $\mathrm{p}<0.01$ \\
\hline $\begin{array}{c}\text { Adiponectin Apn }(\mu \mathrm{g} / \mathrm{ml}) \\
\text { Mean } \pm \text { SD } \\
\text { Range } \\
\end{array}$ & $\begin{array}{c}14.9 \pm 3.9 \\
8-21 \\
\end{array}$ & $\begin{array}{c}7.50 \pm 2.64 \\
4.2-16.8 \\
\end{array}$ & $\mathrm{p}<0.001$ \\
\hline $\begin{array}{c}\text { Nitric oxide NO ( mol/l) } \\
\text { Mean } \pm \text { SD } \\
\text { Range } \\
\end{array}$ & $\begin{array}{c}28.31 \pm 3.57 \\
24.4-40 \\
\end{array}$ & $\begin{array}{c}34.72 \pm 10.20 \\
20.9-61\end{array}$ & $\mathrm{p}<0.001$ \\
\hline $\begin{array}{c}\text { Malondialdehyde MDA ( mol/l) } \\
\text { Mean } \pm \text { SD } \\
\text { Range }\end{array}$ & $\begin{array}{c}0.79 \pm 0.21 \\
0.3-1\end{array}$ & $\begin{array}{c}1.01 \pm 0.26 \\
0.5-1.64\end{array}$ & $\mathrm{p}<0.001$ \\
\hline
\end{tabular}


and MDA ( $\mathrm{p}<0.001)$ in comparison to corresponding levels of controls while plasma levels of adiponectin were significantly lower in type 2 diabetic patients $(\mathrm{p}<0.001)$ in comparison to those of controls.

Table 3 shows demographic and clinical characteristics of obese and non obese diabetic patients in comparison with obese and non obese controls. Plasma levels of glucose, HbA1c were significantly higher in both obese and non obese patients than in obese and non obese controls ( $p<0.001$ for both), plasma levels of cholesterol and triglycerides were significantly higher in both obese patients than in obese controls ( $p<0.01$ and $p<0.001$ respectively) with no significant difference between other subgroups except for triglyceride levels which were significantly higher in obese controls than in nonobese controls $(p<0.05)$. Plasma levels of HDL were significantly lower in obese patients than in obese controls $(\mathrm{p}<0.05)$.

Table 4 shows biochemical parameters of obese and non obese diabetic patients versus obese and non obese controls, where plasma cpeptide levels were significantly higher in obese patients $(p<0.05)$ than those in non obese patients and plasma levels of insulin, cpeptide, NO and MDA were significantly higher in obese patients $(\mathrm{p}<0.01, \mathrm{p}<0.01, \mathrm{p}<$ $0.01, p<0.05$ respectively) while plasma levels of adiponectin were significantly lower in patients $(p<0.001)$ than in obese controls. Plasma levels of insulin were also significantly higher in non obese patients $(p<0.01)$ than in non obese controls while plasma levels of adiponectin were significantly lower in patients $(\mathrm{p}<0.001)$ and plasma adiponectin levels were significantly lower in obese controls $(p<0.05)$ than in non obese controls.

Table 5 illustrates clinical and demographic characteristics of obese diabetic patients with and without cardiovascular diseases (CVD). Glucose and HbA1c levels were significantly higher in both patient subgroups with and without CVD than controls ( $\mathrm{p}<0.001$ for both), cholesterol levels were significantly higher in obese patients with CVD than controls $(p<0.001)$ while significantly higher levels were found in patients with CVD than those without $(p<0.001)$. Triglycerides levels were significantly higher in both patients with and without CVD than controls $(\mathrm{p}<0.001$ and $\mathrm{p}<0.05$ respectively). Patients with CVD showed significantly higher LDL levels and significantly lower HDL levels in comparison to those without CVD and to controls ( $\mathrm{p}<$ 0.001 for $\mathrm{LDL}$ and $\mathrm{p}<0.01$ for HDL).

Table 6 illustrates the plasma levels of main biochemical parameters of type 2 diabetic patients with and without CVD where patients with CVD showed significantly higher levels of insulin and c-peptide than in those of without CVD $(\mathrm{p}<0.01$ and $\mathrm{p}<0.05$ respectively) and in controls ( $p<0.001$ for insulin and $\mathrm{p}<0.01$ for c-peptide), however patients without CVD showed significantly higher levels of these parameters in comparison to those of controls ( $p<0.001$ for both parameters). As regards to plasma levels of adiponectin, significant lower levels were observed in both subgroups compared to controls ( $\mathrm{p}<0.001$ for both subgroups), whereas patients with CVD had significantly lower levels than those without CVD $(\mathrm{p}<0.05)$. NO levels were significantly higher in patients without CVD in comparison to those of controls $(\mathrm{p}<0.01)$ and to patients with CVD $(p<0.05)$. As regards to plasma levels of MDA, higher levels were observed in both subgroups with and without CVD $(\mathrm{p}<0.01)$ compared to controls.

Table 7 shows correlation coefficients (r) of the studied parameters in diabetic patients where adiponectin was significantly positively correlated with HDL ( $p<0.001)$, while it was significantly negatively correlated with each of glucose ( $p<0.001)$, HbA1C $(p<0.001)$, Cholesterol ( $p<0.01)$, LDL $(p<0.001)$, insulin $(p<0.01)$ and c-peptide $(p<0.05)$, also it shows negative correlation with BMI, MDA and triglycerides but didn't reach to a significant level. Insulin was significantly positively correlated with MDA $(\mathrm{p}<0.001)$, glucose $(\mathrm{p}<$ $0.05)$, HbA1C ( $\mathrm{p}<0.05)$, cholesterol ( $\mathrm{p}<$ 0.001), LDL and C-peptide ( $\mathrm{p}<0.001)$ but was significantly negatively correlated with HDL ( $p<0.001)$ and NO ( $<<0.05)$. NO was significantly positively correlated with HDL $(p<0.05)$ but was significantly negatively correlated with glucose $(\mathrm{p}<0.01), \mathrm{HbA1C}$ $(p<0.05)$ on the level of individuals.

Table 8 shows correlation coefficients (r) of the studied parameters in diabetic 
Table 3: Demographic and clinical characteristics of diabetic patients subgrouped according to B.M.I in comparison with controls.

\begin{tabular}{|c|c|c|c|c|c|c|c|c|}
\hline \multirow[b]{2}{*}{ Parameter } & \multicolumn{2}{|c|}{ Controls } & \multicolumn{2}{|c|}{ Patients } & \multicolumn{4}{|c|}{ Significance } \\
\hline & $\begin{array}{l}\text { Obese } \\
n=15\end{array}$ & $\begin{array}{c}\text { Non-obese } \\
n=7\end{array}$ & $\begin{array}{l}\text { Obese } \\
n=40\end{array}$ & $\begin{array}{c}\text { Non-obese } \\
n=11\end{array}$ & $\mathrm{p}^{1}$ & $\mathrm{p}^{2}$ & $\mathrm{p}^{3}$ & $\mathrm{p}^{4}$ \\
\hline $\begin{array}{l}\text { Age (years) } \\
\text { Mean } \pm \text { SD } \\
\text { Range }\end{array}$ & $\begin{array}{c}52.53 \pm 13.7 \\
40-60\end{array}$ & $\begin{array}{c}53.2 \pm 7.6 \\
40-60\end{array}$ & $\begin{array}{c}57.5 \pm 5.4 \\
39-66\end{array}$ & $\begin{array}{c}50.19 \pm 16 \\
40-60\end{array}$ & NS & NS & NS & NS \\
\hline $\begin{array}{c}\mathrm{BMI}\left(\mathrm{Kg} / \mathrm{m}^{2}\right) \\
\text { Mean } \pm \mathrm{SD} \\
\text { Range } \\
\end{array}$ & $\begin{array}{c}31.9 \pm 4.01 \\
26-40\end{array}$ & $\begin{array}{c}22.22 \pm 1.02 \\
21.45-24 \\
\end{array}$ & $\begin{array}{c}30.23 \pm 1.76 \\
26-44\end{array}$ & $\begin{array}{c}23.30 \pm 1.4 \\
22.5-25 \\
\end{array}$ & NS & NS & $p<0.001$ & $p<0.001$ \\
\hline $\begin{array}{c}\text { Glucose }(\mathrm{mg} / \mathrm{dl}) \\
\text { Mean } \pm \text { SD } \\
\text { Range }\end{array}$ & $\begin{array}{c}81.53 \pm 14.2 \\
64-100\end{array}$ & $\begin{array}{c}76.28 \pm 14.8 \\
49-98\end{array}$ & $\begin{array}{c}311.5 \pm 131 \\
134-610\end{array}$ & $\begin{array}{c}279 \pm 114.91 \\
106-500\end{array}$ & $\mathrm{p}<0.001$ & $\mathrm{p}<0.001$ & NS & NS \\
\hline $\begin{array}{c}\mathrm{HbA1c} \% \\
\text { Mean } \pm \text { SD } \\
\text { Range }\end{array}$ & $\begin{array}{c}5.22 \pm 0.34 \\
4.7-6.2 \\
\end{array}$ & $\begin{array}{c}5.39 \pm 0.46 \\
4.9-6.2 \\
\end{array}$ & $\begin{array}{c}9.22 \pm 2.08 \\
6.4-16.4 \\
\end{array}$ & $\begin{array}{c}8.58 \pm 1.89 \\
7-13.5 \\
\end{array}$ & $\mathrm{p}<0.001$ & $\mathrm{p}<0.001$ & NS & NS \\
\hline $\begin{array}{c}\text { Cholesterol } \\
(\mathrm{mg} / \mathrm{dl}) \\
\text { Mean } \pm \mathrm{SD} \\
\text { Range } \\
\end{array}$ & $\begin{array}{c}119.5 \pm 38.7 \\
80-171 \\
\end{array}$ & $\begin{array}{c}112.1 \pm 42.9 \\
80-170 \\
\end{array}$ & $\begin{array}{c}175.9 \pm 62.1 \\
76-350 \\
\end{array}$ & $\begin{array}{c}146.1 \pm 64.7 \\
70-192 \\
\end{array}$ & $\mathrm{p}<0.01$ & NS & NS & NS \\
\hline $\begin{array}{c}\text { TGs }(\mathrm{mg} / \mathrm{dl}) \\
\text { Mean } \pm \text { SD } \\
\text { Range } \\
\end{array}$ & $\begin{array}{c}66.8 \pm 30.49 \\
12-108 \\
\end{array}$ & $\begin{array}{c}60.13 \pm 27.6 \\
10-100 \\
\end{array}$ & $\begin{array}{c}127.4 \pm 52.8 \\
53-275 \\
\end{array}$ & $\begin{array}{c}92.00 \pm 54.4 \\
46-227 \\
\end{array}$ & $p<0.001$ & NS & $\mathrm{p}<0.05$ & NS \\
\hline $\begin{array}{c}\text { LDL }(\mathrm{mg} / \mathrm{dl}) \\
\text { Mean } \pm \text { SD } \\
\text { Range }\end{array}$ & $\begin{array}{c}60.8 \pm 32.03 \\
10-105 \\
\end{array}$ & $\begin{array}{c}59.88 \pm 29 \\
26-104 \\
\end{array}$ & $\begin{array}{c}92.1 \pm 57.76 \\
10-215 \\
\end{array}$ & $\begin{array}{c}85.50 \pm 39.9 \\
14-136 \\
\end{array}$ & NS & NS & NS & NS \\
\hline $\begin{array}{c}\mathrm{HDL}(\mathrm{mg} / \mathrm{dl}) \\
\text { Mean } \pm \mathrm{SD} \\
\text { Range }\end{array}$ & $\begin{array}{c}47.73 \pm 13.8 \\
26-82 \\
\end{array}$ & $\begin{array}{c}45.88 \pm 10.1 \\
33-65 \\
\end{array}$ & $\begin{array}{c}39.78 \pm 11.2 \\
10-65\end{array}$ & $\begin{array}{c}43.25 \pm 8.71 \\
30-62 \\
\end{array}$ & $\mathrm{p}<0.05$ & NS & NS & NS \\
\hline
\end{tabular}

Table 4: Plasma levels of biochemical parameters of diabetic patients subgrouped according to B.M.I in comparison with controls.

\begin{tabular}{|c|c|c|c|c|c|c|c|c|}
\hline \multirow[b]{2}{*}{ Parameter } & \multicolumn{2}{|c|}{ Controls } & \multicolumn{2}{|c|}{ Patients } & \multicolumn{4}{|c|}{ Significance } \\
\hline & $\begin{array}{l}\text { Obese } \\
n=15\end{array}$ & $\begin{array}{c}\text { Non-obese } \\
n=7\end{array}$ & $\begin{array}{l}\text { Obese } \\
n=40\end{array}$ & $\begin{array}{c}\text { Non-obese } \\
n=11\end{array}$ & $\mathrm{p}^{1}$ & $\mathrm{p}^{2}$ & $\mathrm{p}^{3}$ & $p^{4}$ \\
\hline $\begin{array}{c}\text { Insulin }(\mu \mathrm{IU} / \mathrm{ml}) \\
\text { Mean } \pm \text { SD } \\
\text { Range }\end{array}$ & $\begin{array}{c}18.32 \pm 4.32 \\
9-25.5\end{array}$ & $\begin{array}{c}17.69 \pm 4.09 \\
14.6-25.4\end{array}$ & $\begin{array}{c}34.56 \pm 18.1 \\
5.5-85\end{array}$ & $\begin{array}{c}28.63 \pm 6.11 \\
20.5-40.5\end{array}$ & $\mathrm{p}<0.01$ & $\mathrm{p}<0.01$ & NS & NS \\
\hline $\begin{array}{c}\text { C-peptide } \\
\text { (ng/ml) } \\
\text { Mean } \pm \text { SD } \\
\text { Range } \\
\end{array}$ & $\begin{array}{c}1.62 \pm 0.87 \\
0.8-4\end{array}$ & $\begin{array}{c}1.64 \pm 0.40 \\
1-2\end{array}$ & $\begin{array}{l}3.09 \pm 1.07 \\
0.47-4.94 \\
\end{array}$ & $\begin{array}{c}2.22 \pm 1.09 \\
1.1-4.4 \\
\end{array}$ & $\mathrm{p}<0.01$ & NS & NS & $\mathrm{p}<0.05$ \\
\hline $\begin{array}{c}\text { Apn }(\mu \mathrm{g} / \mathrm{ml}) \\
\text { Mean } \pm \text { SD } \\
\text { Range }\end{array}$ & $\begin{array}{c}13.79 \pm 4.08 \\
8-19\end{array}$ & $\begin{array}{c}17.25 \pm 2.1 \\
16-21\end{array}$ & $\begin{array}{c}7.12 \pm 1.49 \\
4.8-9.8\end{array}$ & $\begin{array}{c}7.61 \pm 2.91 \\
4.2-16.8\end{array}$ & $\mathrm{p}<0.001$ & $\mathrm{p}<0.001$ & $\mathrm{p}<0.05$ & NS \\
\hline $\begin{array}{c}\mathrm{NO}(\mu \mathrm{mol} / \mathrm{L}) \\
\text { Mean } \pm \mathrm{SD} \\
\text { Range }\end{array}$ & $\begin{array}{c}28.61 \pm 4.21 \\
24.4-40\end{array}$ & $\begin{array}{c}27.68 \pm 1.84 \\
25.8-30.2\end{array}$ & $\begin{array}{c}36.62 \pm 8.98 \\
20.9-61\end{array}$ & $\begin{array}{c}31.68 \pm 13.7 \\
22.6-59\end{array}$ & $\mathrm{p}<0.01$ & NS & NS & NS \\
\hline $\begin{array}{c}\text { MDA }(\mu \mathrm{mol} / \mathrm{L}) \\
\text { Mean } \pm \text { SD } \\
\text { Range }\end{array}$ & $\begin{array}{c}0.81 \pm 0.2 \\
0.38-1\end{array}$ & $\begin{array}{c}0.73 \pm 0.22 \\
0.3-0.99\end{array}$ & $\begin{array}{c}1.02 \pm 0.28 \\
0.5-1.5\end{array}$ & $\begin{array}{c}0.95 \pm 0.19 \\
0.75-1.6\end{array}$ & $\mathrm{P}<0.05$ & NS & NS & NS \\
\hline
\end{tabular}

$\mathrm{p}^{1}$ is comparison of obese patients with obese controls, $\mathrm{p}^{2}$ is comparison of non obese patients with non obese controls and $\mathrm{p}^{3}$ is comparison between obese control with non obese control and $\mathrm{p}^{4}$ is comparison between obese patient with non obese patient. 
Table 5: Demographic and Clinical characteristics of obese diabetic patients with and without cardiovascular diseases (C.V.D) compared to obese controls.

\begin{tabular}{|c|c|c|c|c|c|c|}
\hline \multirow[b]{2}{*}{ Parameter } & \multirow{2}{*}{$\begin{array}{c}\text { Obese } \\
\text { controls } \\
\mathrm{n}=15 \\
\end{array}$} & \multirow{2}{*}{$\begin{array}{c}\text { Obese patients } \\
\text { with no C.V.D } \\
n=24 \\
\end{array}$} & \multirow{2}{*}{$\begin{array}{c}\text { Obese patients } \\
\text { with C.V.D } \\
n=16 \\
\end{array}$} & \multicolumn{3}{|c|}{ Significance } \\
\hline & & & & $\mathrm{P}^{1}$ & $\mathrm{p}^{2}$ & $\mathrm{p}^{3}$ \\
\hline $\begin{array}{l}\text { Age (years) } \\
\text { Mean } \pm \text { SD } \\
\text { Range }\end{array}$ & $\begin{array}{c}52.53 \pm 13.7 \\
40-60\end{array}$ & $\begin{array}{c}56.9 \pm 7.9 \\
42-66\end{array}$ & $\begin{array}{c}56.5 \pm 6.4 \\
39-62\end{array}$ & NS & NS & NS \\
\hline $\begin{array}{c}\text { BMI }\left(\mathrm{Kg} / \mathrm{m}^{2}\right) \\
\text { Mean } \pm \mathrm{SD} \\
\text { Range }\end{array}$ & $\begin{array}{c}31.9 \pm 4.01 \\
26-40\end{array}$ & $\begin{array}{c}30.25 \pm 3.67 \\
26-41\end{array}$ & $\begin{array}{c}31.89 \pm 4.02 \\
26-44\end{array}$ & NS & NS & NS \\
\hline $\begin{array}{c}\text { Glucose }(\mathrm{mg} / \mathrm{dl}) \\
\text { Mean } \pm \mathrm{SD} \\
\text { Range }\end{array}$ & $\begin{array}{c}81.53 \pm 14.2 \\
64-100 \\
\end{array}$ & $\begin{array}{c}290.56 \pm 135.5 \\
134-600\end{array}$ & $\begin{array}{c}342.64 \pm 120.6 \\
180-610\end{array}$ & $\mathrm{p}^{1}<0.001$ & $\mathrm{p}^{2}<0.001$ & NS \\
\hline $\begin{array}{c}\text { HbA1c } \% \\
\text { Mean } \pm \text { SD } \\
\text { Range }\end{array}$ & $\begin{array}{c}5.21 \pm 0.33 \\
4.7-6.2 \\
\end{array}$ & $\begin{array}{c}8.91 \pm 2.19 \\
6.4-16.4 \\
\end{array}$ & $\begin{array}{c}9.38 \pm 1.72 \\
6.7-13.5 \\
\end{array}$ & $\mathrm{p}^{1}<0.001$ & $\mathrm{p}^{2}<0.001$ & NS \\
\hline $\begin{array}{c}\text { Cholesterol } \\
(\mathrm{mg} / \mathrm{dl}) \\
\text { Mean } \pm \text { SD } \\
\text { Range }\end{array}$ & $\begin{array}{c}119.5 \pm 38.7 \\
80-171\end{array}$ & $\begin{array}{c}144.09 \pm 41.05 \\
70-220\end{array}$ & $\begin{array}{c}218.53 \pm 58.06 \\
140-350 \\
\end{array}$ & NS & $\mathrm{p}^{2}<0.001$ & $\mathrm{p}^{3}<0.001$ \\
\hline $\begin{array}{l}\text { TGs }(\mathrm{mg} / \mathrm{dl}) \\
\text { Mean } \pm \mathrm{SD} \\
\text { Range }\end{array}$ & $\begin{array}{c}66.86 \pm 30.49 \\
12-108 \\
\end{array}$ & $\begin{array}{c}109.47 \pm 52.55 \\
46-250 \\
\end{array}$ & $\begin{array}{c}138.29 \pm 55.74 \\
54-275 \\
\end{array}$ & $\mathrm{p}^{1}<0.05$ & $\mathrm{p}^{2}<0.001$ & NS \\
\hline $\begin{array}{c}\text { LDL }(\mathrm{mg} / \mathrm{dl}) \\
\text { Mean } \pm \text { SD } \\
\text { Range }\end{array}$ & $\begin{array}{c}60.8 \pm 32.03 \\
10-105 \\
\end{array}$ & $\begin{array}{c}74.47 \pm 41.41 \\
10-147\end{array}$ & $\begin{array}{c}135.88 \pm 52.62 \\
52-215\end{array}$ & NS & $\mathrm{p}^{2}<0.001$ & $\begin{array}{l}\mathrm{p}^{3}<0.001 \\
\mathrm{p}^{2}<0.001\end{array}$ \\
\hline $\begin{array}{c}\text { HDL }(\mathrm{mg} / \mathrm{dl}) \\
\text { Mean } \pm \text { SD } \\
\text { Range } \\
\end{array}$ & $\begin{array}{c}47.73 \pm 13.8 \\
26-82 \\
\end{array}$ & $\begin{array}{c}43.75 \pm 10.43 \\
26-65 \\
\end{array}$ & $\begin{array}{c}34.76 \pm 4.32 \\
10-47\end{array}$ & NS & $\mathrm{P}^{2}<0.01$ & $\mathrm{p}^{3}<0.01$ \\
\hline
\end{tabular}

Table 6: Plasma levels of biochemical parameters of obese diabetic patients with and without cardiovascular diseases (C.V.D) compared to obese controls.

\begin{tabular}{|c|c|c|c|c|c|c|}
\hline \multirow[b]{2}{*}{ Parameter } & \multirow{2}{*}{$\begin{array}{c}\text { Obese } \\
\text { controls } \\
n=15\end{array}$} & \multirow{2}{*}{$\begin{array}{c}\text { Obese patients } \\
\text { with no C.V.D } \\
n=24\end{array}$} & \multirow{2}{*}{$\begin{array}{c}\text { Obese patients } \\
\text { with C.V.D } \\
n=16\end{array}$} & \multicolumn{3}{|c|}{ Significance } \\
\hline & & & & $\mathrm{p}^{1}$ & $\mathrm{p}^{2}$ & $\mathrm{p}^{3}$ \\
\hline $\begin{array}{c}\text { Insulin }(\mu \mathrm{IU} / \mathrm{ml}) \\
\text { Mean } \pm \mathrm{SD} \\
\text { Range }\end{array}$ & $\begin{array}{c}18.32 \pm 4.32 \\
9-25.5\end{array}$ & $\begin{array}{c}27.71 \pm 10.48 \\
5.5-62\end{array}$ & $\begin{array}{c}43.82 \pm 20.36 \\
13-85\end{array}$ & $\mathrm{p}^{1}<0.01$ & $\mathrm{p}^{2}<0.001$ & $\mathrm{p}^{3}<0.01$ \\
\hline $\begin{array}{c}\text { C-peptide }(\mathrm{ng} / \mathrm{ml}) \\
\text { Mean } \pm \mathrm{SD} \\
\text { Range }\end{array}$ & $\begin{array}{c}1.62 \pm 0.87 \\
0.8-4\end{array}$ & $\begin{array}{l}2.18 \pm 1.21 \\
0.47-4.94\end{array}$ & $\begin{array}{c}3.19 \pm 1.27 \\
1.2-5\end{array}$ & NS & $\mathrm{p}^{2}<0.01$ & $\mathrm{p}^{3}<0.05$ \\
\hline $\begin{array}{c}\text { Adiponectin }(\mu \mathrm{g} / \mathrm{ml}) \\
\text { Mean } \pm \mathrm{SD} \\
\text { Range }\end{array}$ & $\begin{array}{c}13.79 \pm 4.08 \\
8-19\end{array}$ & $\begin{array}{c}8.21 \pm 2.81 \\
4.2-16.8\end{array}$ & $\begin{array}{c}6.07 \pm 1.50 \\
4.8-11.2\end{array}$ & $\mathrm{p}^{1}<0.001$ & $\mathrm{p}^{2}<0.001$ & $\mathrm{p}^{3}<0.05$ \\
\hline $\begin{array}{c}\text { NO }(\mu \mathrm{mol} / \mathrm{l}) \\
\text { Mean } \pm \mathrm{SD} \\
\text { Range } \\
\end{array}$ & $\begin{array}{c}28.61 \pm 4.21 \\
24.4-40\end{array}$ & $\begin{array}{c}36.93 \pm 10.64 \\
22.6-61 \\
\end{array}$ & $\begin{array}{c}30.33 \pm 7.81 \\
21-48\end{array}$ & $\mathrm{p}^{1}<0.01$ & NS & $\mathrm{p}^{3}<0.05$ \\
\hline $\begin{array}{l}\text { MDA }(\mu \mathrm{mol} / 1) \\
\text { Mean } \pm \text { SD } \\
\text { Range }\end{array}$ & $\begin{array}{c}0.81 \pm 0.2 \\
0.38-1\end{array}$ & $\begin{array}{c}0.95 \pm 0.24 \\
0.5-1.34\end{array}$ & $\begin{array}{c}1.12 \pm 0.28 \\
0.7-1.64\end{array}$ & NS & $\mathrm{p}^{2}<0.01$ & NS \\
\hline
\end{tabular}

$\mathrm{p}^{1}$ is comparison of obese patients without C.V.D and obese controls, $\mathrm{p}^{2}$ is comparison of obese patients with C.V.D and obese controls and $\mathrm{p}^{3}$ is comparison between obese patients with and without CVD. 
Table 7: Correlation coefficient (r) of the studied parameters in diabetic patients.

\begin{tabular}{|c|c|c|c|c|c|c|c|c|c|c|c|c|}
\hline & MDA & Age & BMI & Glucose & $\mathrm{HbA} 1 \mathrm{c}$ & Cholesterol & TGs & LDL & HDL & Insulin & C-peptide & Apn \\
\hline Age & -0.032 & & & & & & & & & & & \\
\hline BMI & 0.136 & -0.048 & & & & & & & & & & \\
\hline Glucose & 0.038 & -0.036 & 0.205 & & & & & & & & & \\
\hline HbA1c & 0.086 & -0.007 & 0.142 & $0.856^{* * *}$ & & & & & & & & \\
\hline Cholesterol & 0.225 & -0.196 & $0.276^{*}$ & 0.227 & $0.277 *$ & & & & & & & \\
\hline TGs & 0.082 & -0.018 & 0.127 & 0.029 & 0.048 & 0.232 & & & & & & \\
\hline LDL & 0.273 & -0.361 & $0.423 * *$ & 0.254 & 0.197 & $0.649 * * *$ & $0.292 *$ & & & & & \\
\hline HDL & 0.071 & -0.008 & -0.196 & $-0.484 * * *$ & $-0.361 *$ & $-0.319 *$ & $-0.380 * *$ & $-0.409 * *$ & & & & \\
\hline Insulin & $0.455^{* * * *}$ & -0.170 & 0.271 & $0.334 *$ & $0.352 *$ & $0.677 * * *$ & 0.123 & $0.553 * * *$ & $-0.320 *$ & & & \\
\hline C-peptide & 0.151 & -0.156 & $0.376^{* *}$ & 0.438 ** & $0.519 * * *$ & $0.523 * * *$ & 0.040 & $0.459 * * *$ & $-0.302 *$ & $0.610 * * *$ & & \\
\hline Apn & -0.196 & 0.167 & -0.151 & $-0.495 * * *$ & $-0.443 * * *$ & $-0.376^{* *}$ & -0.250 & $-0.464 * * *$ & $0.438 * * *$ & $-0.382 * *$ & $-0.353^{*}$ & \\
\hline $\mathrm{NO}$ & -0.074 & 0.138 & 0.085 & $-0.382 * *$ & $-0.326^{*}$ & $-0.319 *$ & -0.075 & -0.237 & 0.299* & $-0.299 *$ & -0.211 & $0.376^{* *}$ \\
\hline
\end{tabular}

Table 8: Correlation coefficient (r) of the studied parameters in diabetic patients with cardiovascular diseases.

\begin{tabular}{|c|c|c|c|c|c|c|c|c|c|c|c|c|}
\hline & MDA & Age & BMI & Glucose & HbA1c & Cholesterol & TGs & LDL & HDL & Insulin & C-peptide & Apn \\
\hline Age & -0.151 & & & & & & & & & & & \\
\hline BMI & 0.397 & -0.186 & & & & & & & & & & \\
\hline Glucose & 0.029 & -0.034 & 0.180 & & & & & & & & & \\
\hline $\mathrm{HbA1c}$ & 0.079 & -0.050 & 0.162 & $0.910 * * *$ & & & & & & & & \\
\hline Cholesterol & 0.276 & -0.110 & 0.109 & 0.235 & 0.453 & & & & & & & \\
\hline TGs & 0.377 & 0.140 & 0.110 & -0.162 & -0.224 & -0.186 & & & & & & \\
\hline LDL & 0.277 & -0.330 & $0.557 *$ & 0.585 & -0.051 & 0.059 & 0.029 & & & & & \\
\hline $\mathrm{HDL}$ & 0.193 & -0.386 & -0.119 & -0.348 & -0.390 & -0.470 & -0.256 & -0.059 & & & & \\
\hline Insulin & $0.539 *$ & -0.265 & 0.193 & 0.349 & 0.419 & $0.617 * *$ & -0.141 & 0.297 & -0.312 & & & \\
\hline C-peptide & 0.379 & 0.009 & 0.371 & 0.463 & $0.549 *$ & 0.481 & -0.146 & 0.230 & -0.256 & $0.753 * * *$ & & \\
\hline Apn & -0.129 & 0.207 & -0.117 & -0.424 & $-0.497 *$ & -0.400 & $-0.598^{*}$ & $-0.405^{*}$ & 0.122 & -0.389 & -0.452 & \\
\hline $\mathrm{NO}$ & 0.291 & 0.101 & 0.397 & -0.221 & -0.178 & -0.178 & -0.078 & -0.243 & 0.295 & -0.289 & -0.325 & 0.215 \\
\hline
\end{tabular}


patients with cardiovascular diseases, where significant positive correlations were found between BMI and LDL ( $\mathrm{p}<0.05), \mathrm{HbA} 1 \mathrm{C}$ and glucose $(\mathrm{p}<0.001), \mathrm{HbA} 1 \mathrm{C}$ and c-peptide $(\mathrm{p}<$ $0.05)$, insulin and MDA ( $\mathrm{p}<0.05)$, insulin and cholesterol $(\mathrm{p}<0.01)$ and insulin with c-peptide $(\mathrm{p}<$ 0.001) while negative significant correlations were observed between adiponectin and $\mathrm{HbA} 1 \mathrm{C}(\mathrm{p}<0.05)$, adiponectin and triglycerides $(r=-0.598, p<0.05)$ and adiponectin with LDL $(\mathrm{p}<0.05)$.

\section{DISCUSSION}

Adiponectin is a collagen-like protein that is solely secreted by adipocytes. Accumulating evidence from animal and human studies demonstrates that adiponectin plays an important role in the pathophysiology of insulin resistance, diabetes, lipid metabolism and inflammation and thus affects risk for cardiovascular disease ${ }^{3}$. The results of the present study showed that plasma levels of adiponectin were significantly lower in type 2 diabetic patients $(\mathrm{p}<0.001)$ in comparison to those of controls. On the other hand fasting blood glucose, glycated hemoglobin, insulin and c-peptide levels were significantly higher in diabetic patients ( $p<0.001$ for each) than control subjects.

These results are in agreement with those reported by Lara-Castro ${ }^{4}$ who have suggested that reduction in plasma adiponectin level may be related the elevation of insulin resistance and/or hyperinsulinemia associated with diabetes. It has been previously reported that adiponectin deficiency contributes to the induction of insulin resistance ${ }^{26}$. This could be explained by the fact that adiponectin stimulated AMP-activated protein kinase (AMPK) phosphorylation and activation, it also increases fatty-acid combustion, glucose uptake, suppressing glucose production from lactate and pyruvate 27 . It has been proposed that adiponectin also increased fatty-acid combustion and energy consumption, in part via Peroxisome Proliferator Activated Receptor alpha (PPAR $\alpha)$ activation, which led to decreased triglyceride content in the liver and skeletal muscle, and thereby a coordinated increase of in-vivo insulin sensitivity ${ }^{28}$. This suggests that adiponectin could potentially be a causative agent for insulin sensitivity ${ }^{29}$. Also overproduction of TNF- $\alpha$ by adipose tissue has been suggested in the development of insulin resistance. Adiponectin interferes with TNF- $\alpha$ signaling in endothelial cells ${ }^{30}$. In the current study, plasma adiponectin levels were significantly inversely correlated with glucose levels $(p<0.001), \mathrm{HbA1c}(\mathrm{p}<0.001)$, insulin $(\mathrm{p}<0.01)$ and $\mathrm{c}$-peptide levels $(\mathrm{p}<0.05)$ in type 2 diabetic patients. These results are in accordance with those previously mentioned reports, and support the view that reduction in plasma adiponectin level may be related to the elevation of insulin resistance in type 2 diabetic patients. As hypoadiponectinemia is frequently associated with insulin resistance, this suggests a cycle during the initial stages of hyperinsulinemia in which high insulin levels lead to a downregulation of adiponectin levels, which in turn decreases insulin sensitivity further, prompting an even higher level of circulating insulin. However the association between adiponectin and insulin resistance have been controversial; whereas some investigators $^{5 \& 6}$ reported that negative significant correlation between plasma adiponectin levels and fasting insulin level, others did not confirm these observations ${ }^{7}$. Although, Zurawska-Klis et al. ${ }^{8}$ did not observe any correlation between plasma level of adiponectin and insulin level, their results revealed significant inverse correlation between plasma adiponectn and HbA1c in type 2 diabetic patients. indicating that the relationship between adiponectin and insulin resistance may be more complex than initially thought.

The association of obesity with development of type 2 diabetes may be partly mediated by altered secretion of adipokines by adipose tissue. Greater adiposity downregulates the secretion of adiponectin ${ }^{31}$. In the present study plasma adiponectin levels of obese and non-obese diabetic patients were significantly lower than those corresponding levels in obese and non-obese controls ( $\mathrm{p}<0.001$ for each). Despite the significant reduction in plasma adiponectin levels of obese controls compared to non-obese ones, there was no significant difference between plasma adiponectin levels of obese and non-obese diabetic patients. However plasma adiponectin levels were non significantly inversely correlated with BMI in all studied groups, and significantly inversely 
correlated with plasma insulin and c-peptide and positively correlated with HDL-C in diabetic patients. These results showed that beside obesity, hyperinsulinemia in the diabetic patients may have been responsible for the decreased plasma adiponectin levels as insulin regulates the secretion of various proteins from adipose tissue ${ }^{32}$. Masaki et al. $^{33}$ demonstrated that the peripheral administration of adiponectin attenuated body weight gain and reduced body adiposity. Consequently attempts to reduce body weight to normalize the plasma adiponectin levels could be effective in preventing the development of diabetes mellitus, atherosclerosis and metabolic syndrome in general in obese individuals ${ }^{34}$.

Atherosclerosis is the major threat to the macrovasculature for patients with and without diabetes. Several metabolic dysfunctions associated with type 2 diabetes have been proposed to play a role in atherosclerosis, including hyperglycemia, formation of advanced glycation end-products (AGE), hyperinsulinemia, endothelial dysfunction, platelet hyperaggregability, coagulation abnormalities, increased oxidative stress and chronic inflammation ${ }^{35}$. The present study showed that plasma adiponectin levels were significantly lower in obese diabetic patients with CVD than those obese without CVD ( $\mathrm{p}<$ $0.05)$.

Similar findings were also reported by Kumuda et $a l^{36}$ who found that plasma levels of adiponectin in diabetic individuals with coronary artery disease (CAD) were lower than in diabetic patients without CAD. Moreover, Pischon et $a l .^{37}$ showed that high plasma adiponectin concentrations were associated with lower risk myocardial infarction (MI) in men, In the current study, increased levels of glucose, HbA1c, insulin and c-peptide were observed in obese diabetic patients with CVD compared to those of obese diabetic patients without cardiovascular disease and in both subgroups of patients compared to controls. Because type 2 diabetes mellitus manifests obesity and hyperglycemia, it is conceived that the proinflammatory state is accentuated. The monocyte-macrophage is a crucial and the most readily accessible cell in the artery wall, secreting several proinflammatory, proatherogenic cytokines such as TNF- $\alpha$, IL-1B and IL6 , which are increased in diabetes ${ }^{38}$.
Besides hypoadiponectinemia and hyperglycemia which were observed in both subgroups of diabetic patients (with and without CVD), a significant inverse correlation was also found between adiponectin and HbA1c levels $(p<0.05)$ in obese diabetic patients with CVD. These results could be explained by the fact that adiponectin suppresses the expression of adhesion molecules, scavenger receptors and TNF- $\alpha$, it may also exert anti-inflammatory effects on glycemia, which may affect circulating cytokine concentrations and lipidemia ${ }^{39}$.

Adiponectin has potential antiatherogenic properties, it strongly inhibits the expression of adhesion molecules, as the intracellular adhesion molecule-1, vascular cellular adhesion molecule-1 and E-selectin, it also inhibits both the production and action of TNF- $\alpha$ that has direct effects on the adhesion molecules Adiponectin also modulates signaling of nuclear factor $\kappa \mathrm{B}(\mathrm{NF \kappa B})$, it also markedly decreases the uptake of oxidized LDL and suppresses macrophage-to-foam cell transformation ${ }^{9}$, Thus, the decreased plasma adiponectin levels in diabetic subjects may play a role in the development of atherosclerotic vascular damage. Another explanation by Hotta et $a l^{32}$ is that accumulation of adiponectin in atherosclerotic vascular walls may accelerate its half-life in plasma, resulting in the reduction of the plasma concentration of adiponectin in subjects with CAD.

Dyslipidemia is highly correlated with atherosclerosis, and almost all patients with diabetes are dyslipidemic ${ }^{40}$. In the present study, significantly increased levels of plasma cholesterol, LDL-c and triglycerides levels were found in obese diabetic patients and obese patients with cardiovascular disease compared to obese controls and obese patients without CVD respectively. Also, plasma adiponectin levels of diabetic patients with or without cardiovascular diseases were inversely correlated with cholesterol, LDL-c and triglyceride levels while were positively correlated with HDL-c levels, Matsuura et al. ${ }^{41}$ found that adiponectin enhanced the mRNA level of apolipoprotein A-I (apoA-I) in cultured human hepatic cells and increased the secretion of apoA-I so increasing HDL assembly. Similar findings were also reported by Zurawska-Klis et $a l^{8}$, who found that plasma adiponectin 
levels were significantly inversely correlated with cholesterol, triglycerides and $\mathrm{HbAlc}$ in type $2 \mathrm{DM}$ patients. Thamer et al. ${ }^{42}$ reported that adiponectin enhances fatty acid oxidation in the circulation and in the skeletal muscle through the activation of AMP kinase, so the accumulation of triglycerides occurs with low levels of adiponectin.

Numerous studies have demonstrated that chronic oxidative stress in diabetic humans and animals, usually related to the metabolism of excess substrates (glucose and fatty acids) present in the hyperglycemic state ${ }^{43}$ as well as to the mitochondrial dysfunction associated with insulin resistance ${ }^{44}$. In the present study diabetic patients showed the state of oxidative stress from their significantly higher levels of lipid peroxides than controls $(\mathrm{p}<0.01)$, also NO levels were significantly higher in diabetic patients than in controls $(\mathrm{p}<0.001)$. These results are in agreement with Wright et al. ${ }^{45}$ and Asl et $a l^{46}$ who reported that hyperglycemia is associated with an increased NO biosynthesis and lipid peroxidation where elevated NO concentrations in subjects with metabolic syndrome and type 2 diabetes were found, on the other hand the results of the present study disagreed with Mahfouz et al. ${ }^{47}$ where serum NO metabolite level was significantly reduced in the both diabetic patient groups compared with controls. Also our results showed that adiponectin was significantly positively correlated with $\mathrm{NO}$ and inversly correlated with MDA. It was reported that adiponectin has vasodilator actions by directly stimulating NO production in endothelial cells using phosphatidylinositol 3kinase pathways involving phosphorylation of endothelial NO synthase (eNOS) by AMPactivated protein kinase (AMPK) $)^{48}$. Adiponectin also enhances NO bioavailability by reducing reactive oxygen species (ROS) production in endothelial cells ${ }^{49}$.

In conclusion the combination of life style modifications and a therapeutic agent that could increase adiponectin levels could be used in treatment of type 2 diabetes, obesity and cardiovascular diseases through improving insulin resistance and preventing atherosclerosis by its anti-inflammatory effects. However, to clarify and be certain of the relationship of adiponectin with these disorders further longitudinal and prospective studies are needed.

\section{REFERENCES}

1- J. Torpy, C. Lynm and R. Glass, "Diabetes", J. Am. Med. Asc., 301, 1620 (2009).

2- E. E. Kershaw and J. S. Flier, "Adipose tissue as an endocrine organ", J. Clin. Endocrinol. Metab., 89 (6), 2548-56 (2004).

3- T. Kadowaki, T. Yamauchi, N. Kubota, K. Hara, K. Ueki and K. Tobe, "Adiponectin and adiponectin receptors in insulin resistance, diabetes, and the metabolic syndrome", J. Clin. Invest., 116 (7). 17841792 (2006).

4- C. Lara-Castro, Y. Fu, B. H. Chung and W. T. Garvey, "Adiponectin and the metabolic syndrome: Mechanisms mediating risk for metabolic and cardiovascular disease", Curr. Opin. Lipidol., 18 (3), 263-70 (2007).

5- K. Esposito, F. Nappo, F. Giugliano, C. Di Palo, M. Ciotola, M. Barbieri, G. Paolisso and D. Giugliano, "Meal modulation of circulating interleukin 18 and adiponectin concentrations in healthy subjects and in patients with type 2 diabetes mellitus", Am. J. Clin. Nutr., 78, 1135-1140 (2003).

6- S. A. Ismail, S. A. Fayed, G. S. Mahmoud and D. B. Abd El-Rehim, "Glucose, insulin and adiponectin relationships in serum of type-2-Egyptian diabetic patients", Res. J. Medicine \& Med. Sci., 4 (2), 241-248 (2009).

7- P. Kleiblova, D. Springer and M. Haluzik, "The influence of hormonal changes during menstrual cycle on serum adiponectin concentrations in healthy women", Physiol. Res., 55, 661-666 (2006).

8- M. Zurawska-Klis, J. Kasznicki, M. Kosmalski, J. Śmigielski and J. Drzewoski, "Adiponectin plasma concentration, type 2 diabetes mellitus, cardiovascular diseases and features of metabolic syndrome", Diabet. Dośw Klin., 9 (2), 81-87 (2009).

9- N. Ouchi, S. Kihara, Y. Arita, M. Nishida, A. Matsuyama, Y. Okamoto, H. Ishigami, M. Kuriyama, K. Kishida, H. Nishizawa, 
K. Hotta, M. Muraguchi, Y. Ohmoto, S. Yamashita, T. Funahashi and Y. Matsuzawa, "Adipocyte-derived plasma protein, adiponectin, suppresses lipid accumulation and class A scavenger receptor expression in human monocytederived macrophages", Circulation, 103, 1057-1063 (2001).

10- Y. Hattori, M. Suzuki, S. Hattori and K. Kasai, "Globular adiponectin upregulates nitric oxide production in vascular endothelial cells", Diabetologia, 46, 15431549 (2003).

11- M. Matsuda, I. Shimomura, M. Sata, Y. Arita, M. Nishida, N. Maeda, M. Kumada, Y. Okamoto, H. Nagaretani, H. Nishizawa, K. Kishida, R. Komuro, N. Ouchi, S. Kihara, R. Nagai, T. Funahashi and Y. Matsuzawa, "Role of adiponectin in preventing vascular stenosis: The missing link of adipo-vascular axis", J. Biol. Chem., 277, 37487-37491 (2002).

12- S. Li, H. Shin, E. Ding and R. van Dam, "Adiponectin levels and risk of type 2 diabetes: A systematic review and metaanalysis", J. Am. Med. Asc., 302 (2), 179188 (2009).

13- World Health Organization (WHO), "Definition, Diagnosis and Classification of Diabetes Mellitus and its Complications: Report of a WHO Consultation", Part 1: Diagnosis and Classification of Diabetes Mellitus (2007).

14- L. Goldman and Ausiello, "Endocrine Disease", In: Silvio E and Robert S. Cecil, "Medicine", $23^{\text {rd }}$ Edition, 2007, pp. 17221748.

15- E. Hu, P. Liang and B. M. Spiegelman, "AdipoQ is a novel adipose-specific gene dysregulated in obesity", J. Biol. Chem., 271, 10697-10703 (1996).

16- J. S. Flier, C. R. Kahn and J. Roth, "Receptors, antireceptor antibodies and mechanisms of insulin resistance", $\mathrm{N}$. Engl. J. Med., 300 (8), 413-419 (1997).

17- R. D. Eastham, "Biochemical Values in Clinical Medicine", $7^{\text {th }}$ Ed., England, John Wright and Sons, Ltd. (1985).

18- P. Trinder, "Determination of blood glucose using 4-Aminophenazone", J. Clin. Path., 22, 246 (1959).

19- E. C. Abraham, "Diabetes", 27, 931 (1978).
20- P. Trinder, Ann. Clin. Biochem., 6, 24 (1969).

21- P. R. Finley et al., Clim. Chem., 24, 391 (1978).

22- G. Assmann, H. U. Jabs, U. Kohnert, W. Nolte and H. Shriewer, Clin. Chim. Acta., 140, 77-83 (1984).

23- A. W. Wahlefeld, In: "Methods of Enzymatic Analysis", Vol. 5, Academic Press, Newyork, 1974, pp. 1831-1835.

24- R. L. Van Bezooijen, I. Que, A. G. Ederveen, H. J. Kloosterboer, S. E. Papapoulos and C. W. Lowik, "Plasma nitrate and nitrite levels are regulated by ovarian steroids but do not correlate with trabecular bone density in rats", J. Endocrinology, 159, 27-34 (1998).

25- W. S. Thayer, "Serum lipid peroxides in rats treated chronically with adriamycin", Biochem. Pharmacol., 33 (14), 2259-2263 (1982).

26- N. Maeda, I. Shimomura, K. Kishida, H. Nishizawa, M. Matsuda, H. Nagaretani, N. Furuyama, H. Kondo, M. Takahashi, Y. Arita, R. Komuro, N. Ouchi, S. Kihara, Y. Tochino, K. Okutomi, M. Horie, S. Takeda, T. Aoyama, T. Funahashi and Y. Matsuzawa, "Diet-induced insulin resistance in mice lacking adiponectin/ ACRP30", Nat. Med., 8, 731-737 (2002).

27- H. Zhou, X. Song, M. Briggs, B. Violand, W. Salsgiver, E. A. Gulve and Y. Luo, "Adiponectin represses gluconeogenesis independent of insulin in hepatocytes", Biochem. Biophys. Res. Commun., 338 (2), 793-9 (2005).

28- T. Yamauchi, J. Kamon, Y. Ito, A. Tsuchida, T. Yokomizo, S. Kita, T. Sugiyama, M. Miyagishi, K. Hara, M. Tsunoda, K. Murakami, T. Ohteki, S. Uchida, S. Takekawa, H. Waki, N. H. Tsuno, Y. Shibata, Y. Terauchi, P. Froguel, K. Tobe, K. Koyasu, K. Taira, T. Kitamura, T. Shimizu, R. Nagai and T. Kadowaki, "Cloning of adiponectin receptors that mediate antidiabetic metabolic effects". Nature, 423 (6941), 762-9 (2003).

29- J. Kawano and R. Arora, "The role of adiponectin in obesity, diabetes, and cardiovascular disease", J. Cardiometab. Syndr., 4 (1), 44-9 (2009). 
30- G. S. Hotamisligil, "Inflammatory pathways and insulin action", Int. J. Obes. Relat. Metab. Disord., 27 (Suppl 3), 53-55 (2003).

31- L. Shanshan, "Adiponectin levels and risk of type 2 diabetes, JAMA, 302 (2), 179188 (2009).

32- K. Hotta, T. Funahashi, Y. Arita, M. Takahashi, M. Matsuda, Y. Okamoto, H. Iwahashi, H. Kuriyama, H. N. Ouchi, K. Maeda, M. Nishida, S. Kihara, N. Sakai, T. Nakajima, K. Hasegawa, M. Muraguchi, Y. Ohmoto, T. Nakamura, S. Yamashita, T. Hanafusa and Y. Matsuzawa, "Plasma concentrations of a novel, adipose-specific protein, adiponectin, in type 2 diabetic patients", Arterioscler. Thromb. Vasc. Biol., 20, 1595-1599 (2000).

33- T. Masaki, S. Chiba, T. Yasuda, T. Tsubone, T. Kakuma, I. Shimomura, T. Funahashi, Y. Matsuzawa and $\mathrm{H}$. Yoshimatsu, "Peripheral, but not central, administration of adiponectin reduces visceral adiposity and upregulates the expression of uncoupling protein in agouti yellow (A $\mathrm{A}^{\mathrm{y} / \mathrm{a})}$ obese mice", Diabetes, 52 (9), 2266-2273 (2003).

34- T. Pischon and E. Rimm, "Adiponectin: A promising marker for cardiovascular disease", Clinical Chemistry, 52, 797-799 (2006).

35- I. J. Goldberg, "Why does diabetes increase atherosclerosis? I don't know!", J. Clin. Invest., 114, 613-5 (2004).

36- M. Kumada, S. Kihara, S. Sumitsuji, T. Kawamoto, S. Matsumoto, N. Ouchi, Y. Arita, Y. Okamoto, I. Shimomura, H. Hiraoka, T. Nakamura and Y. Matsuzawa, "Association of hypoadiponectinemia with coronary artery disease in men", Arterioscler. Thromb. Vasc. Biol., 23, 8589 (2003).

37- T. Pischon, C. J. Girman, G. Hotamisligil, N. Rifai, B. Hu and E. Rimm, "plasma adiponectin levels and risk of myocardial infarction in men", JAMA, 291, 17301737 (2004).

38- G. K. Hansson, "Inflammation, atherosclerosis, and coronary disease", N Engl. J. Med., 352, 1685-1695 (2005).

39- M. B. Schulze, I. Shai, E. B. Rimm, T. Li, N. Rifai and F. B. Hu, "Adiponectin and future coronary heart disease events among men with type 2 diabetes", Diabetes, 54, 534-9 (2006).

40- A. Fagot-Campagna, D. B. Rolka, G. L. Beckles, E. W. Gregg and K. M. Narayan, "Prevalence of lipid abnormalities, awareness, and treatment in US adults with diabetes", ibid., 49 (Suppl. 1), 78 (2000).

41- F. Matsuura, H. Oku, M. Koseki, J. C. Sandoval, M. Yuasa-Kawase, K. Tsubakio-Yamamoto, D. Masuda, N. Maeda, K. Tsuji, M. Ishigami, M. Nishida, K. Hirano, S. Kihara, M. Hori, I. Shimomura and S. Yamashita, Biochem. Biophys. Res. Commun., 358 (4), 10911095 (2007).

42- C. Thamer, J. Machann, O. Tscritter, M. Haap, B. Wietek, D. Dahl, O. Bachmann, A. Fritsche, S. Jacob, M. Stumvoll, F. Schick and H. Haring, "Relationship between serum adiponectin concentration and intramyocellular lipid stores in humans", Horm. Metab. Res., 34, 646-649 (2003).

43- J. Nourooz-Zadeh, A. Rahimi, J. Tajaddini-Sarmadi, H. Tritschler, P. Rosen, B. Halliwell and D. J. Betteridge, "Relationships between plasma measures of oxidative stress and metabolic control in NIDDM", Diabetologia, 40, 647-653 (1997).

44- K. F. Petersen, S. Dufour, D. Befroy, R. Garcia and G. I. Shulman, "Impaired mitochondrial activity in the insulinresistant offspring of patients with type 2 diabetes", N Engl. J. Med., 350, 664-671 (2004).

45- E. Wright, J. L. Scism-Bacon and L. C. Glass, "Oxidative stress in type 2 diabetes: the role of fasting and postprandial glycaemia", Int. J. Clin. Pract., 60 (3), 308-314 (2006).

46- S. Asl, A. Ghasemi and F. Azizi, "Serum nitric oxide metabolites in subjects with metabolic syndrome", Clinical Biochemistry, 41 (16-17), 1342-1347 (2008).

47- M. H. Mahfouz, I. Emara' M. Shouman and M. Ezz, "Asymmetrical dimethylarginine (ADMA) and nitric oxide as potential cardiovascular risk factors in type 2 diabetes mellitus", African Journal of Biochemistry Research, 3 (8), 293-301 (2009). 
48- W. Xi, H. Satoh, H. Kase, K. Suzuki and Y. Hattori, "Stimulated HSP90 binding to eNOS and activation of the PI3-Akt pathway contribute to globular adiponectin-induced NO production: vasorelaxation in response to globular adiponectin", Biochem. Biophys. Res. Commun., 332, 200-205 (2005).
49- H. Motoshima, X. Wu, K. Mahadev and B. J. Goldstein, "Adiponectin suppresses proliferation and superoxide generation and enhances eNOS activity in endothelial cells treated with oxidized LDL", ibid., 315, 264-271 (2004). 